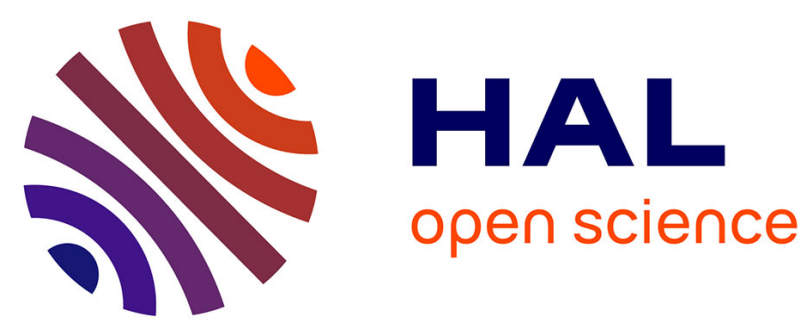

\title{
Pour une archéologie de la pêche sur le littoral Manche-Atlantique de l'Ouest de la France (Âge du bronze, âge du Fer, Antiquité)
}

Marie-Yvane Daire, Vincent Bernard, Cyrille Billard, Océane Charpentier, Yvon Dréano, Louis Dutouquet, Catherine Dupont, Alizée Gabaude, Loïc Langouët, Jean-Paul Le Bihan, et al.

\section{To cite this version:}

Marie-Yvane Daire, Vincent Bernard, Cyrille Billard, Océane Charpentier, Yvon Dréano, et al.. Pour une archéologie de la pêche sur le littoral Manche-Atlantique de l'Ouest de la France (Âge du bronze, âge du Fer, Antiquité). Revue Archéologique de l'Ouest, 2020, 35 (35), pp.199-234. 10.4000/rao.5392 . hal-02486647

\section{HAL Id: hal-02486647 https://hal.science/hal-02486647}

Submitted on 6 Nov 2021

HAL is a multi-disciplinary open access archive for the deposit and dissemination of scientific research documents, whether they are published or not. The documents may come from teaching and research institutions in France or abroad, or from public or private research centers.
L'archive ouverte pluridisciplinaire HAL, est destinée au dépôt et à la diffusion de documents scientifiques de niveau recherche, publiés ou non, émanant des établissements d'enseignement et de recherche français ou étrangers, des laboratoires publics ou privés. 


\section{OpenEdition} Journals

SEARCH Tout OpenEdition

\section{Revue archéologique de \\ l'Ouest}

$35 \mid 2018$

Varia

\section{Pour une archéologie de la pêche sur le littoral Manche- Atlantique de l'ouest de la France (Âge du bronze, âge du Fer, Antiquité)}

Towards an archaeology of fishing along the channel and atlantic coasts of western France (Bronze age, iron age and roman period)

Für eine Archäologie der Fischerei an der westfranzösischen Atlantik- und Kanalküste: Bronzezeit - Eisenzeit und römische Epoche

Por una arqueología de la pesca en el litoral de la Mancha y el Atlántico en el oeste de Francia Edad del Bronce, Edad del Hierro, Antigüedad

Marie-YVAne Daire, Vincent Bernard, CYrille Billard, OcÉANE Charpentier, Yvon DrÉANo, LOUIS DutOUQueT, CATHERINE DuPONT, AlizÉE GABAUde, LoÏC LANGOUËT†', JEAN-PAUl LE BiHAN, Caroline Mougne, Alain Provost, Jean-François Villard ET LAURENT QUESNEL

p. $199-234$

https://doi.org/10.4000/rao.5392

\section{Résumés}


Français English Deutsch Español

L'archéologie de la pêche souffre encore d'un manque de visibilité dans l'Ouest de la France, malgré le formidable potentiel offert par les littoraux de la Manche et de l'Atlantique en termes d'exploitation des ressources marines et côtières. Cet article rassemble une multitude de données jusqu'ici très dispersées dans les publications, rapports de fouilles, notices, voire d'objets inédits ; celles-ci touchent aux structures, vestiges fauniques et instruments de pêche dans un domaine géographique englobant la Normandie, la Bretagne et les Pays-de-la-Loire et sur un spectre chronologique couvrant la Protohistoire récente (âges du Bronze et du Fer) et l'Antiquité. La discussion s'enrichit du caractère interdisciplinaire de l'approche et aborde l'évolution des techniques, les choix de collecte, le rôle de la pêche dans l'économie de subsistance... Cette première synthèse démontre enfin que l'archéologie de la pêche constitue un sujet de recherche à part entière, sujet appelé à se développer dans un avenir proche.

The archaeology of fishing suffers from a lack of visibility in the west of France, in spite of the potential for the study of the exploitation of marine and costal resources in the Channel and on the Atlantic Coast. This paper brings together data that was until now dispersed in publications, site reports, and short papers and uses unpublished objects that relate to features, zooarchaeology and fishing equipment found in Normandy, Brittany and the Pays-de-la-Loire for the Metal Ages and the Roman period. The discussion is enhanced by its interdisciplinary character and covers subjects such as the evolution of techniques, the fishing choices made, the role of fishing in subsistence economies. This first overview finally shows that the archaeology of fishing constitutes an important research topic, which we hope to develop in the future.

Bei der Archäologie der Fischerei ist in Westfrankreich weiterhin ein ungenügender Forschungsstand zu beklagen, trotz des ausgezeichneten Potentials, das die Atlantik- und die Kanalküste für Studien zur Nutzung mariner und littoraler Ressourcen bieten. Dieser Beitrag versammelt zahlreiche Forschungsergebnisse, die bisher auf eine Vielzahl verschiedener Publikationen, Grabungsberichte und Fundnotizen verstreut waren; ferner behandelt er auch bisher unveröffentlichte Funde. Die Ergebnisse stützen sich auf Befunde, archäozoologisches Fundmaterial sowie auf Gerätschaften des Fischfangs aus einem geographisch weit gesteckten Gebiet, das die Normandie, die Bretagne, und die Region Pays de la Loire umfasst und einen chronologischen Rahmen von den Metallzeiten bis zur römischen Zeit umspannt. Die Diskussion der Ergebnisse profitiert hierbei insbesondere von einem interdisziplinären Forschungsansatz. Es werden die technologische Entwicklung, die Auswahl der genutzten Ressourcen sowie die Stellung der Fischerei bei der Selbstversorgung behandelt. Diese erste Synthese soll ferner unterstreichen, dass eine Archäologie der Fischerei ein wichtiges eigenständiges Forschungsfeld darstellt, dass es in naher Zukunft weiter zu entwickeln gilt.

La arqueología de la pesca sufre aún de una falta de visibilidad en el oeste de Francia, a pesar del potencial extraordinario del litoral del Canal de la Mancha y el Atlántico en términos de explotación de recursos marinos y costeros. Este artículo reúne una gran cantidad de datos hasta ahora ampliamente dispersos en publicaciones, informes de excavaciones, notas e incluso objetos inéditos, relacionados con estructuras, restos de fauna e instrumentos de pesca en un área geográfica que abarca Normandía, Bretaña y los Países del Loira y en un espectro cronológico que incluye la Protohistoria reciente (Edad del Bronce y del Hierro) y la Antigüedad. La discusión se ve enriquecida por la naturaleza pluridisciplinar del enfoque y se aborda la evolución de las técnicas, la selección en la recolección, el papel de la pesca en la economía de subsistencia... Esta primera síntesis finalmente demuestra que la arqueología de la pesca constituye plenamente un tema de investigación, un tema que probablemente se desarrolle en un futuro próximo.

\section{Entrées d'index}

Mots-clés : pêche, ressources, littoral, mer, protohistoire, Antiquité, ouest de la France

Keywords: fishing, resources, coasts, sea, Metal Ages, Antiquity, Western France

Schlagwortindex: fischerei, ressourcen, küste, meer, metallzeiten, römische zeit, westfrankreich

Indice de palabras clave: pesca, recursos, litoral, Oeste de Francia, Protohistoria, Antigüedad

\section{Extrait du texte}




\section{Aperçu du texte}

\section{Introduction}

Les systèmes qui découlent de l'activité de pêche peuvent être groupés en trois catégories : systèmes d'acquisition, de transformation et de consommation qui intéragissent avec d'autres systèmes techniques. L'exploitation des poissons marins peut dépendre de différents facteurs environnementaux, comme les caractéristiques biologiques des proies et du milieu dans lequel elles évoluent, mais aussi des facteurs sociaux et techniques tels que les technologies employées ou les formes d'organisations sociales auxquelles les pêcheurs appartiennent (McGoodwin, 2003).

Les techniques de pêche sont ainsi adaptées aux écosystèmes marins exploités, mais aussi aux espèces recherchées, et sont souvent l'aboutissement d'enseignements cumulés et d'une expérience considérable. L'organisation des sociétés, leur structure, et le but de l'opération (personnelle ou commerciale) sont aussi des ...

\section{Pour citer cet article}

Référence papier Marie-Yvane Daire, Vincent Bernard, Cyrille Billard, Océane Charpentier, Yvon Dréano, Louis Dutouquet, Catherine Dupont, Alizée Gabaude, Loïc Langouët†, Jean-Paul Le Bihan, Caroline Mougne, Alain Provost, Jean-François Villard et Laurent Quesnel, « Pour une archéologie de la pêche sur le littoral Manche-Atlantique de l'ouest de la France (Âge du bronze, âge du Fer, Antiquité) ", Revue archéologique de l'Ouest, 35 | 2018, 199-234.

Référence électronique Marie-Yvane Daire, Vincent Bernard, Cyrille Billard, Océane Charpentier, Yvon Dréano, Louis Dutouquet, Catherine Dupont, Alizée Gabaude, Loïc Langouët†, Jean-Paul Le Bihan, Caroline Mougne, Alain Provost, Jean-François Villard et Laurent Quesnel, « Pour une archéologie de la pêche sur le littoral Manche-Atlantique de l'ouest de la France (Âge du bronze, âge du Fer, Antiquité) ", Revue archéologique de l'Ouest [En ligne], 35 | 2018, mis en ligne le 12 février 2022, consulté le 04 janvier 2021. URL : http://journals.openedition.org/rao/5392 ; DOI : https://doi.org $/ 10.4000 /$ rao.5392

\section{Auteurs}

Marie-Yvane Daire

CNRS, UMR 6566 CReAAH, Centre de recherche en Archéologie, Archéosciences, Histoire, Rennes.

Articles du même auteur

Le tumulus de Kervingar (Plouarzel, Finistère) : un regard nouveau sur les débuts de la recherche archéologique d'après-guerre en Bretagne [Texte intégral]

The Kervingar tumulus (Plouarzel, Finistère): a new look at the beginnings of archaeological research in post-war Brittany

Der Grabhügel von Kervingar (Plouarzel, Finistère): Neue Betrachtungen zu den Anfängen archäologischer Forschung in der Nachkriegszeit in der Bretagne

El túmulo Keringar (Plouarzel, Finistère): una nueva mirada en los inicios de la investigación arqueológica en el momento de la posguerra en Bretaña

Paru dans Revue archéologique de l'Ouest, 32 | 2015

Théodore Monod (1902-2000) et l'archéologie bretonne : note sur un épisode méconnu de la vie du « fou du désert » [Texte intégral]

Théodore Monod (1902-2000) and Breton archaeology: notes on a little known episode of the life of "the madman of the desert" 
conocido de la vida del "loco del desierto"

Paru dans Revue archéologique de l'Ouest, 30 | 2013

Terre de pêcheries [Texte intégral]

Paru dans Revue archéologique de l'Ouest, 30 | 2013

Salz-Reich. 7000 Jahre Hallstatt [Texte intégral]

Paru dans Revue archéologique de l'Ouest, 26 | 2009

Des Gaulois sur l'île Guennoc (Landéda, Finistère) [Texte intégral]

Gaulish people on Guennoc Island (Landéda, Finistère)

Paru dans Revue archéologique de l'Ouest, 25 | 2008

Vincent Bernard

CNRS, UMR 6566 CReAAH, Centre de recherche en Archéologie, Archéosciences, Histoire,

Rennes.

Articles du même auteur

Le site de La Rochette à Mauron (Morbihan) : les multiples occupations d'un promontoire [Texte intégral]

The site of La Rochette at Mauron (Morbihan) : Multiple Occupations of a Promontory

Paru dans Revue archéologique de l'Ouest, 28 | 2011

Cyrille Billard

DRAC SRA Normandie et UMR 6566 CReAAH, Centre de recherche en Archéologie,

Archéosciences, Histoire, Rennes.

Articles du même auteur

Le site Bronze ancien de La Caillouerie à Saint-Lô d'Ourville (50), un site spécialisé en contexte littoral ? [Texte intégral]

The early Bronze Age settlement of "La Caillouerie" in Saint-Lô d'Ourville (50), a specialized site in seashore environment?

Der älterbronzezeitliche Fundplatz "La Caillouerie" von Saint-Lô d'Ourville (Manche), ein spezialisierter Fundplatz im Küstenbereich?

El sitio de la Edad de Bronce Antigua de "La Caillouerie" en Saint-Lô-d'Ourville (Manche): un sitio especializado en contexto costero?

Paru dans Revue archéologique de l'Ouest, 31 | 2014

Trois sépultures atypiques du Second âge du Fer à Reviers (Calvados) [Texte intégral] Three atypical Iron Age graves from Reviers (Calvados)

Paru dans Revue archéologique de l'Ouest, 26 | 2009

Les occupations du Mésolithique final de Biéville-Beuville « Le Vivier » (Calvados) [Texte intégral]

The "Le Vivier" final Mesolithic occupations at Biéville-Beuville (Calvados)

Paru dans Revue archéologique de l'Ouest, 25 | 2008

Le monument funéraire néolithique de Poses « Sur la Mare » (Eure) [Texte intégral]

Paru dans Revue archéologique de l'Ouest, 23 | 2006

G. Marchand et A. Tresset, Unité et diversité des processus de néolithisation sur la façade atlantique de l'Europe (6e-4e millénaire avant J.-C.) [Texte intégral]

Paru dans Revue archéologique de l'Ouest, 23 | 2006

Océane Charpentier

Master Université de Rennes 2.

Yvon Dréano

CRAVO.

Louis Dutouquet

HELP et AMARAI (Association Manche Atlantique pour la Recherche Archéologique dans les Îles).

Catherine Dupont

CNRS, UMR 6566 CReAAH, Centre de recherche en Archéologie, Archéosciences, Histoire, Rennes.

Articles du même auteur 
de l'âge du bronze aux Batardières (Saint-Vincent-sur-Jard, Vendée) [Texte intégral] Indices of marine resources at the end of the 2nd millennium BC: the fireplace of the bronze age to Batardières (Saint-Vincent-sur-Jard, Vendée)

Índices de explotación de los recursos marinos a finales del segundo milenio antes de Cristo: el hogar de la Edad de Bronce en Batardières (Saint-Vincent-sur-Jard, Vendée)

Hinweise auf die Ausbeutung mariner Ressourcen am Ende des 2. Jahrtausends v. Chr.: die bronzezeitliche Feuerstelle bei Batardières (Saint-Vincent-sur-Jard, Vendée, Frankreich)

Paru dans Revue archéologique de l'Ouest, 32 | 2015

Alizée Gabaude

Master Université de Rennes 1.

Loïc Langouët†

CeRAA (Centre Régional d'Archéologie d'Alet) et AMARAI (Association Manche Atlantique pour la Recherche Archéologique dans les Îles).

Jean-Paul Le Bihan

CRAF (Centre de Recherche Archéologique du Finistère), Quimper.

Caroline Mougne

MNHN, Paris et UMR 6566 CReAAH, Centre de recherche en Archéologie, Archéosciences, Histoire, Rennes.

\author{
Alain Provost \\ Indépendant. \\ Jean-François Villard \\ CRAF (Centre de Recherche Archéologique du Finistère), Quimper.
}

Articles du même auteur

Un établissement agricole et artisanal de la fin du haut Moyen Âge au 22 de la rue Bourg-les-Bourgs à Quimper (Finistère) [Texte intégral]

Excavation on an early medieval rural settlement and workshop at 22 rue Bourg-les-Bourgs, Quimper (Finistère)

Paru dans Revue archéologique de l'Ouest, 28 | 2011

Le dépôt de l'âge du Bronze moyen de Keravel en Lannilis (Finistère) [Texte intégral] The Keravel Middle Bronze Age Hoard, Lannilis (Finistère)

Paru dans Revue archéologique de l'Ouest, 26 | 2009

La nécropole à crémations du Hallstatt final-La Tène ancienne de Kerjaouen en Quimper (Finistère) [Texte intégral]

Paru dans Revue archéologique de l'Ouest, 23 | 2006

Laurent Quesnel

CNRS, UMR 6566 CReAAH, Centre de recherche en Archéologie, Archéosciences, Histoire,

Rennes.

Articles du même auteur

Le site de Kermenguy à Châteauneuf-du-Faou (Finistère) [Texte intégral disponible en février 2022]

Des vestiges d'habitats du Néolithique moyen et de l'âge du Bronze ancien, une fosse de la fin du IIle millénaire

The site of Kermenguy at Châteauneuf-du-Faou (Finistère). Remains of Middle Neolithic and Early Bronze age settlements and a pit of the end of the third millenium

Paru dans Revue archéologique de l'Ouest, 35 | 2018

Le tumulus de Kervingar (Plouarzel, Finistère) : un regard nouveau sur les débuts de la recherche archéologique d'après-guerre en Bretagne [Texte intégral]

The Kervingar tumulus (Plouarzel, Finistère): a new look at the beginnings of archaeological research in post-war Brittany

Der Grabhügel von Kervingar (Plouarzel, Finistère): Neue Betrachtungen zu den Anfängen archäologischer Forschung in der Nachkriegszeit in der Bretagne

El túmulo Keringar (Plouarzel, Finistère): una nueva mirada en los inicios de la investigación arqueológica en el momento de la posguerra en Bretaña

Paru dans Revue archéologique de l'Ouest, 32 | 2015 
The site of La Rochette at Mauron (Morbihan) : Multiple Occupations of a Promontory

Paru dans Revue archéologique de l'Ouest, 28 | 2011

Des Gaulois sur l'île Guennoc (Landéda, Finistère) [Texte intégral]

Gaulish people on Guennoc Island (Landéda, Finistère)

Paru dans Revue archéologique de l'Ouest, 25 | 2008

\section{Droits d'auteur}

@ Presses universitaires de Rennes 